\title{
Metastable surface oxide on CoGa(100): Structure and stability
}

\author{
A. Vlad, ${ }^{1}$ A. Stierle, ${ }^{1, *, \dagger}$ M. Marsman, ${ }^{2}$ G. Kresse, ${ }^{2}$ I. Costina,,${ }^{1,}$ H. Dosch,,${ }^{1,3}$ M. Schmid,${ }^{4}$ and P. Varga ${ }^{4}$ \\ ${ }^{1}$ Max-Planck Institut für Metallforschung, D-70569 Stuttgart, Germany \\ ${ }^{2}$ Computational Materials Physics and Center for Computational Materials Science, Universität Wien, A-1090 Wien, Austria \\ ${ }^{3}$ Deutsches Elektronen-Synchrotron (DESY), D-22603 Hamburg, Germany \\ ${ }^{4}$ Institut für Angewandte Physik, Technische Universität Wien, A-1040 Wien, Austria
}

(Received 28 November 2009; revised manuscript received 29 January 2010; published 2 March 2010)

\begin{abstract}
We investigated the structure and formation of a surface oxide and bulk $\beta-\mathrm{Ga}_{2} \mathrm{O}_{3}$ on $\mathrm{CoGa}(100)$ from ultrahigh vacuum to 1 bar oxygen pressure in a temperature range from 300 to $1040 \mathrm{~K}$. We combined in situ surface $\mathrm{x}$-ray diffraction with scanning tunneling microscopy, atomic force microscopy, and density-functional theory calculations. We find that the two-dimensional epitaxial surface oxide layer exhibits a $p 2 \mathrm{~mm}$ symmetry with an additional mirror plane as compared to the bulk oxide. The surface oxide layer is found to form under metastable conditions at an oxygen chemical potential $\sim 1.6 \mathrm{eV}$ above the stability limit for bulk $\beta-\mathrm{Ga}_{2} \mathrm{O}_{3}$. The formation of the bulk oxide is kinetically hindered by the presence of the oxygen-terminated surface oxide, which most likely hampers dissociative oxygen chemisorption. We observe that below $620 \mathrm{~K}$, the surface oxide is surprisingly stable at 1 bar oxygen pressure. Substrate faceting accompanies the bulk oxide formation at temperatures higher than $1020 \mathrm{~K}$.
\end{abstract}

DOI: 10.1103/PhysRevB.81.115402

PACS number(s): 81.16.Pr, 61.05.cf, 68.37.Ef, 71.15.Mb

\section{INTRODUCTION}

Oxidation of metals and metallic alloys is of crucial importance for different technological applications such as heterogeneous catalysis, microelectronic devices, gas sensors, and high-temperature resistant coatings. In general, in situ investigations of the oxide stability at industrially relevant conditions of temperature and oxygen pressure, as well as a precise structural and chemical analysis of the oxide layers are desired, in order to have a fundamental understanding of the processes governing the aforementioned applications. In this context, the limited applicability of the conventional surface science methods under high-pressure environments led to the emergence of the so-called "pressure gap," an issue closely related to heterogeneous catalysis but also important for fundamental studies on oxide stability on metallic systems. Sustained scientific efforts have been recently made in order to close the pressure gap: an important tool for getting insight into this problem is the combination of different experimental methods with ab initio thermodynamics. Many of the reported studies were conducted on oxidation of transition metals since these systems are highly important in heterogeneous catalysis. Ultrathin oxides (also called surface oxides) composed of an oxygen ion double layer were found to form on late $4 d$ transition-metal surfaces, ${ }^{1}$ showing a remarkable stability against transformation to bulk oxides. The formation of surface oxides is not restricted to noble-metal surfaces: ultrathin, epitaxial oxide layers with a thickness in the subnanometer regime, and a well-defined interface to the substrate form under controlled conditions by the selective oxidation of alloys, i.e., $\mathrm{NiAl},{ }^{2-8} \mathrm{FeAl},{ }^{9} \mathrm{CoGa},{ }^{10,11}$ and $\mathrm{Cu}-\mathrm{Al} .^{12}$ Such oxide films are used as insulating barriers in magnetic tunnel junctions ${ }^{13}$ and are known to play a critical role in the device performance. For instance, spin-dependent electron tunneling was shown to occur through a gallium oxide layer used as an insulating tunnel barrier. ${ }^{14}$ Besides having the desired properties, requirements for practical ap- plications include a homogeneous chemical composition, thickness in the $1 \mathrm{~nm}$ regime, as well as stability and reproducibility in preparation of the oxide layer. Ultimately, the optimization of device performance renders the investigation of the structure and stability of the oxide layers mandatory.

The present study focuses on the oxide formation on the $\mathrm{CoGa}(100)$ surface by varying the temperature from 300 to $1100 \mathrm{~K}$ and oxygen partial pressures ranging from ultrahigh vacuum (UHV) to 1 bar. Stoichiometric $\mathrm{CoGa}-$ an intermetallic B2-ordered alloy-exhibits selective oxidation of $\mathrm{Ga}$ when exposed to oxygen environments. Bulk $\mathrm{Ga}_{2} \mathrm{O}_{3}$ exists in different phases based on close-packed oxygen lattices. ${ }^{15,16}$ $\beta-\mathrm{Ga}_{2} \mathrm{O}_{3}$ is the only known thermodynamically stable bulk phase of Ga oxide. ${ }^{10}$ It has a monoclinic structure as depicted in Fig. 1(a) with oxygen ions forming a distorted fcc lattice and $\mathrm{Ga}$ atoms occupying octahedral and tetrahedral sites.

Formation of a crystalline ultrathin gallium oxide film on $\mathrm{CoGa}(100)$ was previously observed upon exposure at relatively low oxygen partial pressures and temperatures higher than $550 \mathrm{~K} .{ }^{18,19}$ The atomistic structure and the growth mechanism of the ultrathin oxide layer formed during the initial stages of $\mathrm{CoGa}(100)$ oxidation have been recently discussed. ${ }^{11}$

In the present work we give additional information on the structural refinement of the ultrathin gallium oxide layer based on surface x-ray diffraction (SXRD), scanning tunneling microscopy (STM), and density-functional theory (DFT) calculations, including local bonding configurations, symmetry, and STM image simulation. We find that the surface oxide structure is compatible with $p 2 \mathrm{~mm}$ symmetry, differing from $\mathrm{Ga}_{2} \mathrm{O}_{3}$ bulk oxide exhibiting only one mirror plane (space group $\mathrm{C} 2 / \mathrm{m}$ ).

In addition, we followed the transition from the surface oxide to bulk $\beta-\mathrm{Ga}_{2} \mathrm{O}_{3}$ as a function of temperature $T$ and oxygen partial pressure $p$ by means of in situ surface x-ray diffraction measurements. In this way, the oxide $(p-T)$ stability diagram was mapped out and compared to results from $a b$ initio thermodynamics. We find that the formation of the sur- 


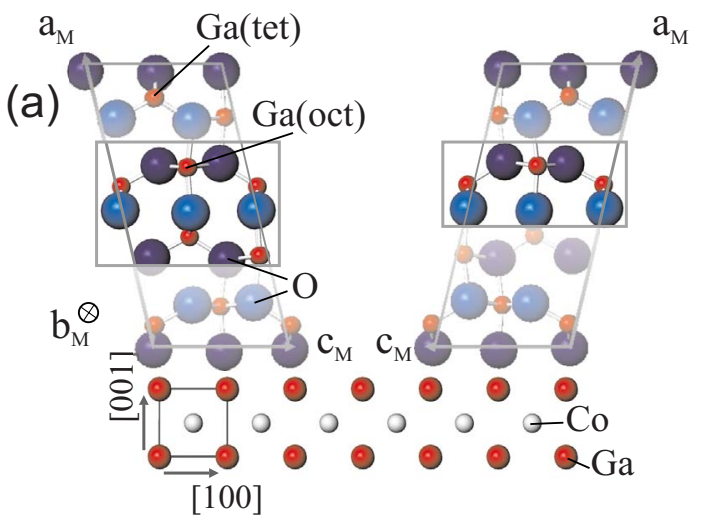

(b)

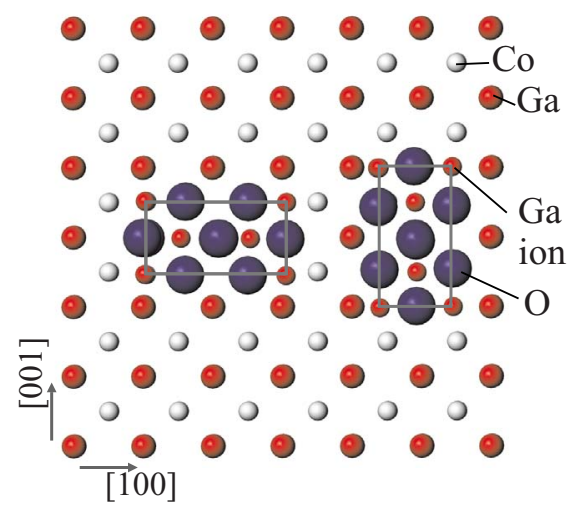

(c)

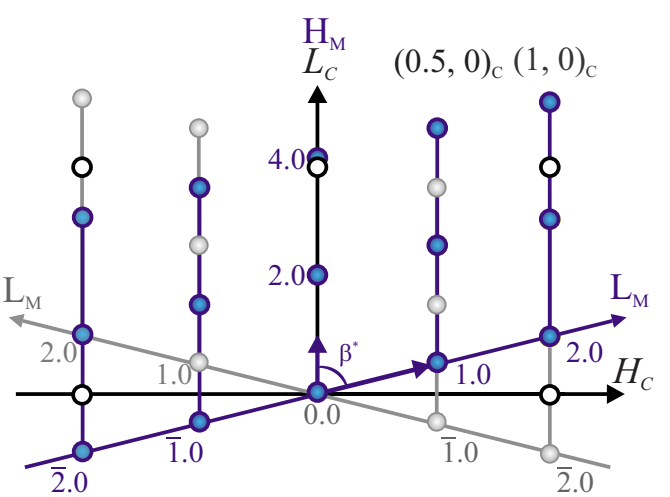

FIG. 1. (Color online) Schematic representation of the $\mathrm{CoGa}(100)$ substrate and possible arrangement of the $\mathrm{Ga}_{2} \mathrm{O}_{3}$ bulk structure on it. (a) Side view of two $\mathrm{Ga}_{2} \mathrm{O}_{3}$ mirror domains. (b) Top view of two rotational domains. The bulk structure is made up by a stacking of Ga ions in octahedral (oct) and tetrahedral (tet) coordination within the distorted fcc oxygen sublattice. The lattice constants of $\beta-\mathrm{Ga}_{2} \mathrm{O}_{3}$ are at $273.2 \mathrm{~K}: a_{\mathrm{M}}=12.214 \AA, b_{\mathrm{M}}=3.037 \AA$, and $c_{\mathrm{M}}=5.798 \AA$ and $\alpha=90^{\circ}, \beta=103.83^{\circ}$, and $\gamma=90^{\circ}$ (Ref. 17). [The "M" subscripts indicate the monoclinic coordinates]. (c) Outof-plane reciprocal $(H, 0, L)$ plane of $\mathrm{CoGa}$ (black open circles). Superimposed are the reflections arising from the two mirror domains of $\beta-\mathrm{Ga}_{2} \mathrm{O}_{3}$, indicated by the dark gray (blue) and light gray circles.

face oxide layer takes place under metastable equilibrium conditions at an oxygen chemical potential considerably above the one for $\mathrm{Ga}_{2} \mathrm{O}_{3}$ bulk oxide formation. The formation of the bulk oxide, in turn, is governed by strong kinetic barriers, which most prominently manifest themselves in the stability of the surface oxide layer at 1 bar oxygen pressure at room temperature. At temperatures above $1000 \mathrm{~K}$ the $\mathrm{CoGa}(100)$ surface undergoes oxidation-induced faceting, as revealed by in situ SXRD and ex situ atomic force microscopy (AFM) experiments.

\section{EXPERIMENTAL AND COMPUTATIONAL DETAILS}

A nominally $\mathrm{Co}_{50} \mathrm{Ga}_{50}$ single crystal with a diameter of 8 $\mathrm{mm}$ and a thickness of $2 \mathrm{~mm}$ was used for all the experiments presented here. The specimen was cut and polished parallel to the (100) planes and oriented by x-ray diffraction better than $0.05^{\circ}$. The main impurities of the CoGa sample are carbon, oxygen, and sulfur. After several oxidationdesorption and sputtering-annealing cycles, no impurities could be detected by means of Auger electron spectroscopy measurements.

The crystal truncation rod (CTR) measurements for the surface oxide structure determination were performed in an in situ UHV chamber ${ }^{20}$ at beamline ID32 at the European Synchrotron Radiation Facility, Grenoble using an incident photon energy of $20 \mathrm{keV}$. The CTR data were background subtracted and standard correction factors were applied. ${ }^{21} \mathrm{~A}$ complete structural analysis was made by measuring 16 nonsymmetry-equivalent surface rods and four independent crystal truncation rods (in total 850 independent structure factors). The simultaneous fit of the surface and crystal truncation rods was performed using the software package ROD. ${ }^{22}$ Relaxations of the atoms in $x, y$, and $z$ directions were allowed conserving $p 2 \mathrm{~mm}$ symmetry. For the fit, an equal distribution of $(2 \times 1)$ and $(1 \times 2)$ domains was assumed. The in situ near atmospheric pressure oxidation measurements were carried out in a portable UHV-high-pressure compatible diffraction chamber at the MPI-MF beamline ${ }^{23}$ at Angströmquelle Karlsruhe using a photon energy of $10 \mathrm{keV}$. The sample temperature was controlled by a ChromelAlumel type $\mathrm{K}$ thermocouple spot welded to its side. The oxygen pressure was determined by a Pirani gauge above 5 $\times 10^{-3}$ mbar and by a cold cathode gauge at lower pressures. Above $5 \times 10^{-3}$ mbar the chamber was isolated from the turbo molecular pump and the experiments were performed under static conditions. The STM measurements were performed at room temperature using a customized commercial STM (Omicron $\mu$-STM). The STM images were obtained in Vienna using constant current mode and an electrochemically etched tungsten tip conditioned by sputtering with $\mathrm{Ar}^{+}$under UHV conditions. The AFM measurements were performed in contact mode at ambient conditions.

The computational investigations on the surface oxide structural stability were performed using the Vienna $a b$ initio simulation package, ${ }^{24}$ employing the projector-augmentedwave method m,26 $^{25}$ and the local-density approximation of Ceperley and Alder. ${ }^{27,28}$ The valence wave functions were expanded in a plane-wave basis set with a kinetic-energy cutoff of $400 \mathrm{eV}$. Brillouin-zone integration was performed using grids of $(5 \times 9 \times 1) k$ points in the $(2 \times 1)$ surface cell for all calculations. The CoGa bulk lattice constant obtained with this setup is $a_{0}=2.82 \AA$ and the bulk $\beta-\mathrm{Ga}_{2} \mathrm{O}_{3}$ lattice parameters $a=12.15 \AA, b=3.02 \AA, c=5.76 \AA$, and $\beta$ $=103.8^{\circ}$, in good agreement with the experimental results. 
The CoGa(100) substrate was modeled by a slab of four Co and four $\mathrm{Ga}(2 \times 1)$ layers. The bottom four layers were kept fixed, whereas the top four layers were allowed to relax. The oxide layers were placed on top of the relaxed side of the slab (asymmetric setup). Periodic images were separated by at least $10 \AA$ of vacuum.

STM image simulations were based on the TersoffHamann model. ${ }^{29-31}$ In this model the STM tip is described by a $s$-type wave function, tip-substrate interactions are neglected and the tunneling process is treated within a perturbational approach. Hence the tunneling current is proportional to the charge density of sample states at the position of the tip apex atom, including only states that satisfy elastic tunneling conditions corresponding to the applied tip-sample bias voltage. In accordance with the experimental tip polarity and bias voltage, the density of (occupied) states was integrated from $-50 \mathrm{meV}$ up to the Fermi level.

\section{RESULTS}

\section{A. Surface oxide structure determination}

For the surface oxide structure determination, an ultrathin oxide layer was prepared by oxidation at $723 \mathrm{~K}$ and 5 $\times 10^{-7}$ mbar $\mathrm{O}_{2}$ for $480 \mathrm{~s}$. The two-dimensional surface oxide grows epitaxially on the substrate and forms a $(2 \times 1)$ superstructure in two domains rotated by $90^{\circ}, 18,19$ as indicated in Figs. 1(a) and 1(b).

As a starting model for determining the structure, we have taken $\beta-\mathrm{Ga}_{2} \mathrm{O}_{3}$ because its lattice match to the CoGa substrate opens up the possibility of epitaxial growth: $b_{\mathrm{M}}$ possesses a misfit of $+5.5 \%$ and $c_{\mathrm{M}} / 2$ a misfit of $+0.7 \%$ with respect to the $\mathrm{CoGa}(100)$ surface with $a_{\mathrm{C}}=2.878 \AA$ (the subscript " $\mathrm{M}$ " stands for the monoclinic $\beta-\mathrm{Ga}_{2} \mathrm{O}_{3}$ structure). The $b_{\mathrm{M}}$ and $c_{\mathrm{M}}$ axes are in the surface plane and oriented parallel to the $\mathrm{CoGa}(010)$ and $\mathrm{CoGa}(100)$ directions, respectively, building up the $(2 \times 1)$ superstructure. The $a_{\mathrm{M}}$ axis is tilted by $14^{\circ}$ with respect to the surface normal and in principle two types of out-of-plane domains for each in-plane domain exist: the oxide unit cell can be rotated by $180^{\circ}$ around the surface normal or mirrored, still fulfilling the epitaxial relationships described above, as shown in Fig. 1(a). It must be noted, however, that the $14^{\circ}$ tilt of the unit cell is only a consequence of finding the smallest unit cell; the oxygen anions in $\beta-\mathrm{Ga}_{2} \mathrm{O}_{3}$ form a distorted fcc(100) lattice showing only negligible tilt $\left(0.1^{\circ}\right)$. The primitive, nonorthorhombic unit cell, and the existence of mirror domains are caused by the distribution of octrahedral and tetrahadral Ga sites in the cell, which does not exhibit mirror symmetry with respect to a plane perpendicular to the $c_{\mathrm{M}}$ axis.

The x-ray diffraction data are presented using the reciprocal lattice of the CoGa substrate. The out-of-plane reciprocal $\left(\begin{array}{lll}H & 0 & L\end{array}\right)$ plane of $\mathrm{CoGa}$ (black open circles) together with the reflections arising from the two mirror domains of $\beta$ - $\mathrm{Ga}_{2} \mathrm{O}_{3}$ (indicated by the blue and gray circles) are shown in Fig. 1(c). (The subscript " $\mathrm{C}$ " indicates the cubic coordinates). Due to the $(2 \times 1)$ periodicity, the oxide reciprocal lattice exhibits additional rods of scattered intensity at noninteger $H$ and $K$ values, the so-called surface rods. At integer $H$ and $K$ values all oxide domains contribute and the crystal
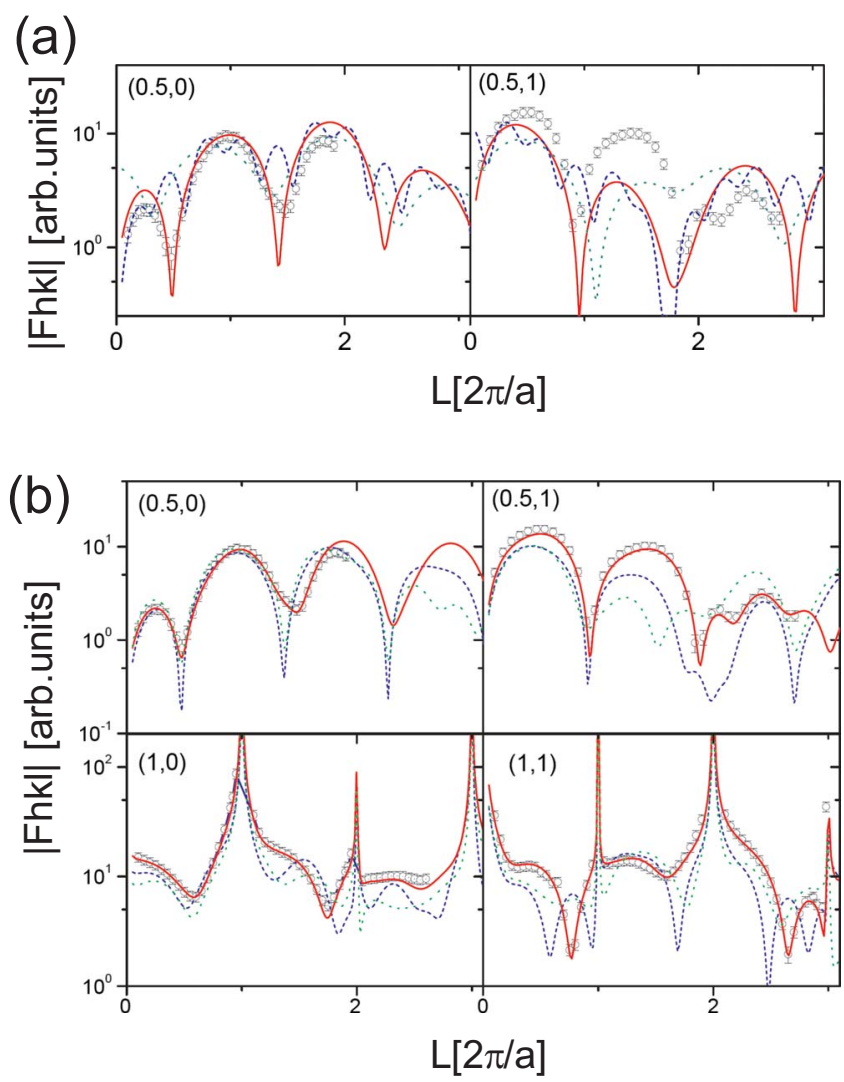

FIG. 2. (Color online) (a) Structure factor amplitude calculated for two surface rods- $(0.5,0)$ and $(0.5,1)$ - corresponding to one $\mathrm{Ga}_{2} \mathrm{O}_{3}$ unit cell (dashed, blue line), for a five (solid, red line), and a three layers slab (dotted, green line) as highlighted in Fig. 1(a). The experimental structure factors as a function of the reciprocal-lattice coordinate, $L$, perpendicular to the surface for different in-plane momentum transfers $(H, K)$ are indicated by the black open circles. (b) Experimental (black symbols) and calculated (solid, red curves) structure factors for the best-fit structure as a function of the reciprocal-lattice coordinate, $L$. Out of the complete data set, two surface rods and two CTRs are plotted. For comparison, the structure factors calculated for two other DFT-relaxed structures (dashed (blue) and dotted (green) lines) are plotted (see text for details).

truncation rods contain information on the registry of the overlayer with respect to the substrate while the surface rods are only sensitive to the oxide structure.

As a first step, the finite film thickness oscillation period on the surface rods was used to determine the thickness of the oxide layer. Structure factors were calculated for a full bulk unit cell and for slabs with different thickness and compared to the data, as shown in Fig. 2(a). The slabs used for calculations are highlighted in Fig. 1(a) [five layers slab $\left(\mathrm{OGa}^{\text {oct }} \mathrm{O}-\mathrm{Ga}^{\text {tet }}-\mathrm{O}-\mathrm{Ga}^{\text {tet }}-\mathrm{OGa}{ }^{\text {oct }} \mathrm{O}\right)$ - left rectangle and three layers slab $\left(\mathrm{OGa}^{\text {oct }} \mathrm{O}-\mathrm{Ga}^{\text {tet }}-\mathrm{O}\right)$-right rectangle]. We note that the structure factors calculated for the five layers thick slab match the period of the oscillations on the surface rods best.

Second, all possible slabs with the same thickness and both $\mathrm{Ga}$ and Co substrate terminations were considered for the x-ray and DFT refinement. The structures were relaxed and structure factors of configurations characterized by a DFT energy minimum were compared with the diffraction 

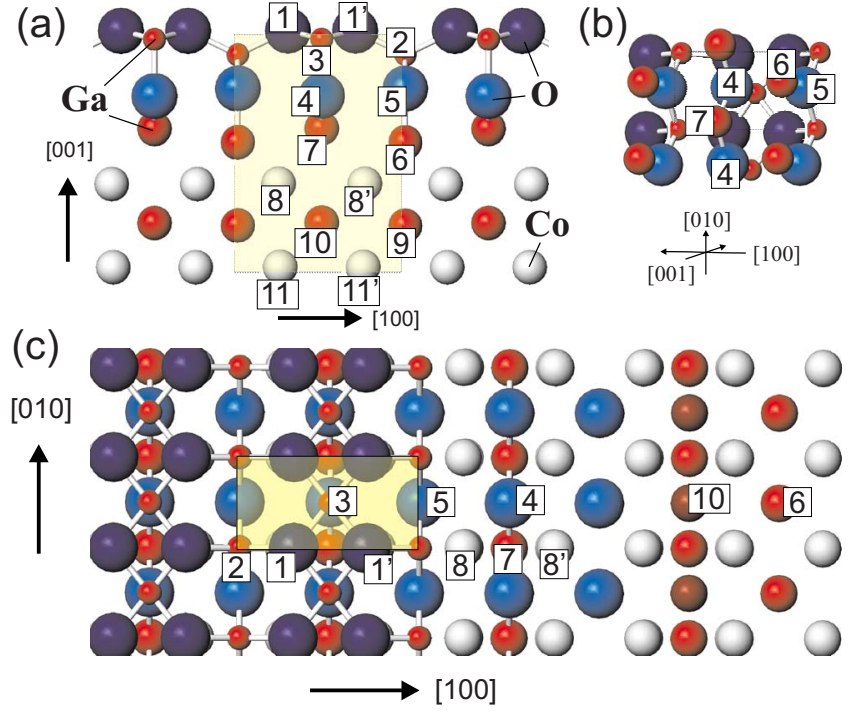

FIG. 3. (Color online) (a) Side view, (b) detailed bottom view of the bonding configuration, and (c) top view of the best-fit surface oxide model [see Tables I and II]. The oxygen atoms located close to the interface are represented by the light gray (blue) spheres for clarity.

data. As an example, Fig. 2(b) shows a comparison between the experimental $\mathrm{x}$-ray diffraction structure factors (black symbols) and the calculated structure factors for two DFTrelaxed structures with $\mathrm{Ga}$ and $\mathrm{Co}$ interfacial termination. The two models were derived from the five layers slab highlighted in Fig. 1(a) having bulk stoichiometry. However, the comparison between the experimental and calculated structure factors makes the poor agreement immediately apparent [see Fig. 2(b)].

The best fit to the data $\left(\chi^{2}=0.96\right)$ was achieved for the structural model shown in Figs. 3(a)-3(c), only allowing relaxations that obey $p 2 \mathrm{~mm}$ symmetry and including two domains rotated by $90^{\circ}$ as shown in Fig. 1(b). The finite thickness of the ultrathin film and its position on high-symmetry sites of the substrate introduces a second mirror plane as compared to the bulk structure. This model was derived from the above-mentioned five-layer model by a complete removal of the interfacial oxygen ion layer. It consists of an oxygen ion double layer similar to two fcc(100) layers; Ga ions at the surface occupy truncated octahedral and tetrahedral sites, reflecting the structural elements of $\beta-\mathrm{Ga}_{2} \mathrm{O}_{3}$ (Fig. $3)$. At the interface, $\mathrm{Ga}$ ions occupy hollow and bridge sites of the Co-terminated substrate, which results in a pronounced buckling of the interfacial Ga layer. The lower (hollow-site) interfacial Ga ions bind to an oxygen ion directly above; the higher (bridge) ones are between two $\mathrm{O}$ ions; thus the buckling of the oxygen layer above the interfacial $\mathrm{Ga}$ is the inverse of the Ga below.

The average $\mathrm{Ga}^{\text {tet }} \mathrm{O}$ distance is $1.88 \AA$ and the $\mathrm{O}-\mathrm{Ga}^{\text {tet }} \mathrm{O}$ angles are in the range $102.0^{\circ}-128.5^{\circ}$, indicating distorted tetrahedral coordination geometry. The average $\mathrm{Ga}^{\text {oct }}-\mathrm{O}$ distance is $1.89 \AA$ and the $\mathrm{O}-\mathrm{Ga}^{\text {oct }}$-O angles are in the range $76.5^{\circ}-100.6^{\circ}$. These values compare well to those corresponding to $\beta-\mathrm{Ga}_{2} \mathrm{O}_{3}$ : $\mathrm{Ga}^{\text {tet }}-\mathrm{O}$ and $\mathrm{Ga}^{\text {oct }}-\mathrm{O}$ distances are 1.84 and $1.99 \AA$, respectively, and the $\mathrm{O}-\mathrm{Ga}^{\text {tet }}-\mathrm{O}$ and $\mathrm{O}-\mathrm{Ga}^{\text {oct }}-\mathrm{O}$
TABLE I. Bond lengths of the surface oxide vs bulk $\beta-\mathrm{Ga}_{2} \mathrm{O}_{3}$. The bonds are labeled according to Figs. 3(b) and 3(c).

\begin{tabular}{ccc}
\hline \hline Bond & Surface oxide $(\AA)$ & Bulk oxide $(\AA)$ \\
\hline $3-1$ & 1.87 & 1.94 \\
$3-1^{\prime}$ & 1.87 & 2.07 \\
$3-4$ & 1.96 & 1.94 \\
$2-1$ & 1.91 & 1.83 \\
$2-5$ & 1.85 & 1.83 \\
$2-1^{\prime}$ & 1.91 & 1.86 \\
$6-5$ & 1.85 & 1.94 \\
$7-4$ & 1.82 & 1.83 \\
\hline \hline
\end{tabular}

angles are in the range $105.9^{\circ}-119.6^{\circ}$ and $80.9^{\circ}-94.1^{\circ}$, respectively. ${ }^{17} \mathrm{~A}$ direct comparison between the bond lengths and angles of the bulk and the surface oxide is given in Tables I and II, respectively. The bonds and angles are labeled according to Figs. 3(b) and 3(c). All oxygen atoms in the surface oxide have bonds to three Ga ions in an almost planar configuration. Also in the bulk oxide, most $\mathrm{O}$ ions have three bonds but some have four.

The layer sequence and stoichiometry of the ultrathin oxide cell is $\mathrm{O}_{2}-\mathrm{Ga}_{2}-\mathrm{O}_{2}-\mathrm{Ga}_{2}$, different from the $\mathrm{Ga}_{2} \mathrm{O}_{3}$ bulk stoichiometry. Similar to the ultrathin alumina films, ${ }^{4}$ we must consider the interfacial Ga layer partly metallic; a charge-neutral film is obtained if we assign a formal valency of +1 to these $\mathrm{Ga}$ atoms.

Because of the low x-ray scattering contrast between Co and $\mathrm{Ga}$, it is not directly possible to distinguish between $\mathrm{Co}$ or Ga termination at the interface from the fit of the X-ray data. However, the DFT-relaxed structure with Ga substrate termination below the interfacial $\mathrm{Ga}$ layer is by $0.94 \mathrm{eV} /$ oxygen atom less favorable than the one with Co termination. This is in line with the obvious property of the ordered $\mathrm{CoGa}$ alloy that $\mathrm{Co}-\mathrm{Ga}$ bonds are favored with respect to $\mathrm{Co}-\mathrm{Co}$ and $\mathrm{Ga}-\mathrm{Ga}$ bonds. Comparing the experimental (SXRD) and calculated (DFT) atomic positions, we find remarkably good agreement, with only slight indications of underbinding in DFT evidenced by the larger interlayer distances in the oxide layer (Table III).

TABLE II. O-Ga-O bond angles of the surface oxide vs bulk $\beta-\mathrm{Ga}_{2} \mathrm{O}_{3}$. The angles are labeled according to Figs. 3(b) and 3(c); "oct" and "tet" stand for octahedral and tetrahedral coordination of $\mathrm{Ga}$, "int" denotes $\mathrm{Ga}$ at the interface.

\begin{tabular}{cccc}
\hline \hline Angle & Ga coordination & $\begin{array}{c}\text { Surface oxide } \\
(\mathrm{deg})\end{array}$ & $\begin{array}{c}\text { Bulk oxide } \\
(\mathrm{deg})\end{array}$ \\
\hline $1-3-1^{\prime}$ & oct & 76.5 & 80.9 \\
$1-3-1$ & oct & 100.6 & 94.1 \\
$1-2-5$ & tet & 105.9 & 105.9 \\
$5-2-5$ & tet & 102.0 & 111.9 \\
$1^{\prime}-2-1$ & tet & 128.5 & 119.6 \\
$1^{\prime}-2-5$ & tet & 105.9 & 106.8 \\
$4-7-4$ & int & 104.1 & 119.9 \\
\hline \hline
\end{tabular}


TABLE III. Atomic positions of the surface oxide, in fractional coordinates with respect to unit cell marked by the shaded (yellow) rectangle in Fig. $3[a=5.756 \AA$ and $b=c=2.878 \AA]$.

\begin{tabular}{cccccccc}
\hline \hline Atom label & Atom type & $x_{\exp }$ & $x_{\text {DFT }}$ & $y_{\exp }$ & $y_{\text {DFT }}$ & $z_{\exp }$ & $z_{\text {DFT }}$ \\
\hline $11^{\prime}$ & $\mathrm{Co}$ & 0.753 & 0.753 & 0.500 & 0.500 & -0.504 & -0.505 \\
11 & $\mathrm{Co}$ & 0.247 & 0.247 & 0.500 & 0.500 & -0.504 & -0.505 \\
10 & $\mathrm{Ga}$ & 0.500 & 0.500 & 0.000 & 0.000 & -0.007 & -0.015 \\
9 & $\mathrm{Ga}$ & 0.000 & 0.000 & 0.000 & 0.000 & 0.026 & 0.023 \\
$8^{\prime}$ & $\mathrm{Co}$ & 0.744 & 0.743 & 0.500 & 0.500 & 0.493 & 0.485 \\
8 & $\mathrm{Co}$ & 0.256 & 0.257 & 0.500 & 0.500 & 0.493 & 0.485 \\
7 & $\mathrm{Ga}$ & 0.500 & 0.500 & 0.000 & 0.000 & 0.988 & 0.975 \\
6 & $\mathrm{Ga}$ & 0.000 & 0.000 & 0.500 & 0.500 & 1.152 & 1.143 \\
5 & $\mathrm{O}$ & 0.000 & 0.000 & 0.000 & 0.000 & 1.544 & 1.576 \\
4 & $\mathrm{O}$ & 0.500 & 0.500 & 0.000 & 0.000 & 1.631 & 1.643 \\
3 & $\mathrm{Ga}$ & 0.500 & 0.500 & 0.500 & 0.500 & 2.036 & 2.053 \\
2 & $\mathrm{Ga}$ & 0.000 & 0.000 & 0.000 & 0.000 & 2.223 & 2.246 \\
$1^{\prime}$ & $\mathrm{O}$ & 0.799 & 0.779 & 0.500 & 0.500 & 2.325 & 2.377 \\
1 & $\mathrm{O}$ & 0.201 & 0.221 & 0.500 & 0.500 & 2.325 & 2.377 \\
\hline \hline
\end{tabular}

To further corroborate the structural model, we have performed high-resolution STM measurements. Figure 4(a) shows an atomically resolved overview image, exhibiting almost perfect $(1 \times 1)$ structure with a lattice constant of about $2.87 \AA$. A comparison with the simulated STM image for the structure described above exhibits good agreement [see Fig. 4(b) upper panel]. As observed for other oxygen-terminated surface oxides, $1,4,8$ the topmost oxygen ions appear as protrusions (bright). They are only slightly displaced toward to each other by $0.17 \AA$ along the [100] direction according to the DFT-determined structure $(0.28 \AA$ according to SXRD;

(a)

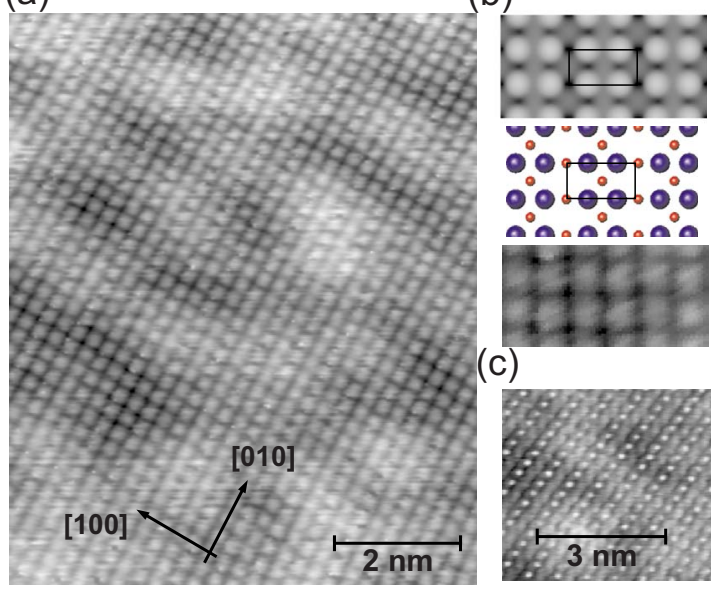

FIG. 4. (Color online) (a) STM image of the surface oxide on CoGa(100) obtained at a sample voltage of $-49 \mathrm{mV}$ and a tunnel current of $0.3 \mathrm{nA}$ imaging occupied states. (b) Simulated STM image (upper panel), structural model for the topmost O-Ga ion layer and the Ga ion layer below the surface (middle panel), and zoom in the experimental STM image in (a) (lower panel). Small spheres correspond to Ga ions, large spheres to oxygen ions. (c) STM image obtained under similar tunneling conditions exhibiting a much stronger $(2 \times 1)$ contrast. here we consider DFT more accurate because oxygen is a weak scatterer for $x$ rays). In some STM images a much stronger $(2 \times 1)$ contrast was observed, with alternating bright and dark oxygen rows [Fig. 4(c)]; the same was also reported in a previous study. ${ }^{10}$ This type of image is not reproduced by the STM image simulations and must be therefore explained by an asymmetric tip of the STM.

\section{B. Transition from surface oxide to bulk $\beta-\mathrm{Ga}_{2} \mathrm{O}_{3}$}

The stability diagram of the gallium oxide and its formation on $\mathrm{CoGa}(100)$ from room temperature to temperatures up to $1043 \mathrm{~K}$, was mapped out by means of in situ SXRD measurements. The surface oxide formation was observed as follows: the CoGa(100) crystal was first heated at a chosen temperature and subsequently exposed to oxygen. The intensity evolution was followed in real time on an surface oxidesensitive reciprocal space position, i.e. $(0,0.5,0.96)$, which corresponds to a maximum of intensity along the $(0,0.5, L)$ surface oxide rod. The $\mathrm{O}_{2}$ pressure was subsequently increased in time steps of about $600 \mathrm{~s}$.

To test the stability of the surface oxide layer toward bulk oxide formation, a fresh surface oxide layer was prepared for each temperature at $723 \mathrm{~K}$ and $2 \times 10^{-7}$ mbar oxygen pressure for $1800 \mathrm{~s}$. Subsequently, oxygen was applied at different pressures while the sample was held at temperatures between room temperature and $923 \mathrm{~K}$. The transformation of the surface oxide layer was monitored by following the intensity of a bulk oxide-sensitive reciprocal space position at $(0.5,0,1.08)$ and subsequently performing $L$ scans. This point in reciprocal space corresponds to the $(401)_{\mathrm{M}}$ Bragg peak of $\beta-\mathrm{Ga}_{2} \mathrm{O}_{3}$.

The stability conditions for surface or bulk gallium oxide at different temperatures and partial oxygen pressures as observed experimentally are indicated in Fig. 5. The white area marks the regime in which the clean surface or a chemisorbed oxygen phase is stable (not determined), whereas the 


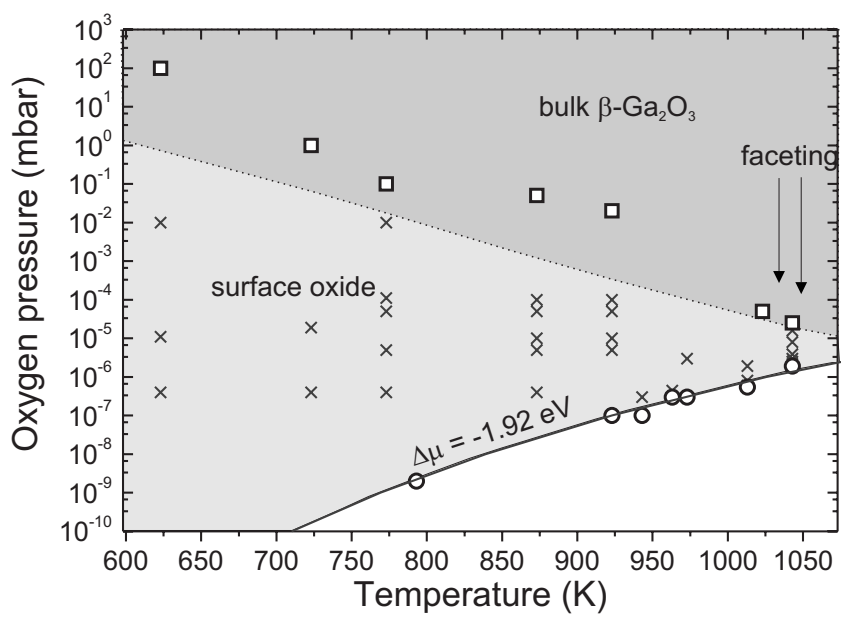

FIG. 5. Experimental ( $p-T)$ stability diagram of gallium oxide formation on $\mathrm{CoGa}(100)$. The open circles and rectangles indicate the onset of surface and the bulk oxide formation, respectively, whereas the crosses mark the conditions at which the surface oxide was found to be stable. The solid line represents a constant oxygen chemical potential of $\Delta \mu_{\mathrm{O}}=-1.92 \mathrm{eV}$. Note that the $\Delta \mu$ line calculated for the stability limit of the bulk oxide lies about 20 orders of magnitude in pressure below. The dotted line is a guide to the eye for the transition from the surface oxide to the bulk oxide.

light and the dark gray areas correspond to the surface and bulk gallium oxide stability region, respectively.

\section{Surface oxide formation}

Depending on the temperature, the surface oxide was found to form at oxygen pressures between $2 \times 10^{-9}$ and $2.6 \times 10^{-6}$ mbar, as indicated in Fig. 5. In the temperature range between 790 and $1045 \mathrm{~K}$ the formation/decomposition of the surface oxide was found to be reversible while the oxygen pressure was increased or decreased, respectively. These $(T, p)$ data points of the transition can be used to obtain the oxygen chemical potential $\mu_{\mathrm{O}}(T, p)$, above which the surface oxide gets more stable than an oxygen chemisorption phase which very likely exists at lower oxygen surface coverage (not investigated here). ${ }^{32}$ The solid line in Fig. 5 is a fit to the data corresponding to $\Delta \mu_{\mathrm{O}}(T, p)=-1.92 \mathrm{eV}$. In addition, the decomposition of the surface oxide layer was observed at $793 \mathrm{~K}$ under UHV conditions (residual pressure 2 $\times 10^{-9}$ mbar, partially water vapor), consistent with this value of the oxygen chemical potential.

\section{Stability of surface oxide and bulk oxide formation}

First we discuss the stability of the surface oxide layer at temperatures below $623 \mathrm{~K}$. The room-temperature results are given as an example here. The $(0.5,0)$ surface oxide rod is plotted in Fig. 6(a). Upon oxygen exposure up to 1 bar, no significant change in the surface oxide signal takes place, indicating a high kinetic stability of the surface oxide layer.

At $623 \mathrm{~K}$ the formation of very thin bulk oxide islands was observed after one hour exposure at 0.1 bar $\mathrm{O}_{2}$ (scan not shown here). The diffraction signal along the $(0.5,0)$ rod changed much more rapidly as the temperature for the oxy-
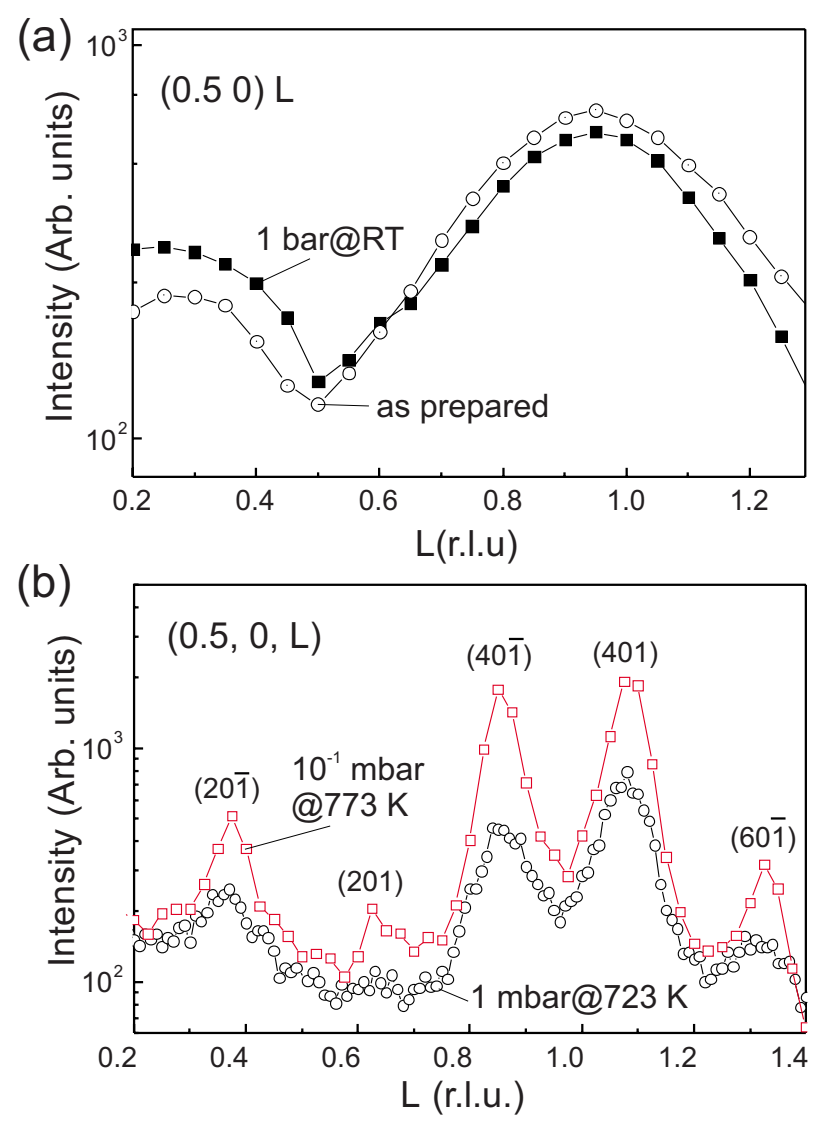

FIG. 6. (Color online) (a) Diffracted intensity along the $(0.5,0)$ rod for the as-prepared surface oxide layer (open circles) and after exposure to 1 bar of oxygen at room temperature (filled squares). (b) Diffracted intensity along the $(0.5,0, L)$ reciprocal-lattice direction after bulk oxide growth at $723 \mathrm{~K}$ (open (black) circles) and 773 $\mathrm{K}$ [open (red) squares].

gen exposure of the surface oxide layer was increased to 723 $\mathrm{K}$ and above. Several peaks appeared along the $(0.5,0)$ rod at $L=0.37,0.61,0.85,1.09$, and 1.33, as plotted in Fig. 6(b). These reflections can be indexed in monoclinic bulk $\beta-\mathrm{Ga}_{2} \mathrm{O}_{3}$ coordinates. Rocking scans (with the rotation axis perpendicular to the surface) through the $(401)_{\mathrm{M}}$ oxide peak (not shown here) exhibit a distinct maximum with a full width at half maximum of $5.5^{\circ}$, indicative for the epitaxial growth of a defective layer.

The monoclinic $\vec{a}^{*}$ axis points out of the surface and the bulk oxide exhibits a commensurate $(2 \times 1)$ superstructure in the surface plane; in spite of the $5.5 \%$ misifit, commensurability was still observed at a bulk oxide thickness of $3 \mathrm{~nm}$. We always find pairs of reflections with indices $\left(\begin{array}{lll}H & 0 & L\end{array}\right)_{\mathrm{M}}$ and $\left(\begin{array}{lll}H & 0 & \bar{L}\end{array}\right)_{\mathrm{M}}$, giving evidence for bulk oxide mirror domains as pictured in Fig. 1(a). Since no finite-thickness oscillations are observed along the $(0.5,0)$ rod, the oxide surface must be rather rough. From the width of the $(401)_{M}$ and $(40 \overline{1})_{M}$ reflections an average saturation oxide film thickness of about $3.1 \mathrm{~nm}$ at $723 \mathrm{~K}$ and $4.4 \mathrm{~nm}$ at $773 \mathrm{~K}$ is determined.

Similar experiments were performed at different temperatures between 623 and $1043 \mathrm{~K}$ and the corresponding oxygen pressures for the onset of bulk oxide growth range from $10^{2}$ to $2.5 \times 10^{-5}$ mbar, as plotted in Fig. 5. With increasing oxi- 

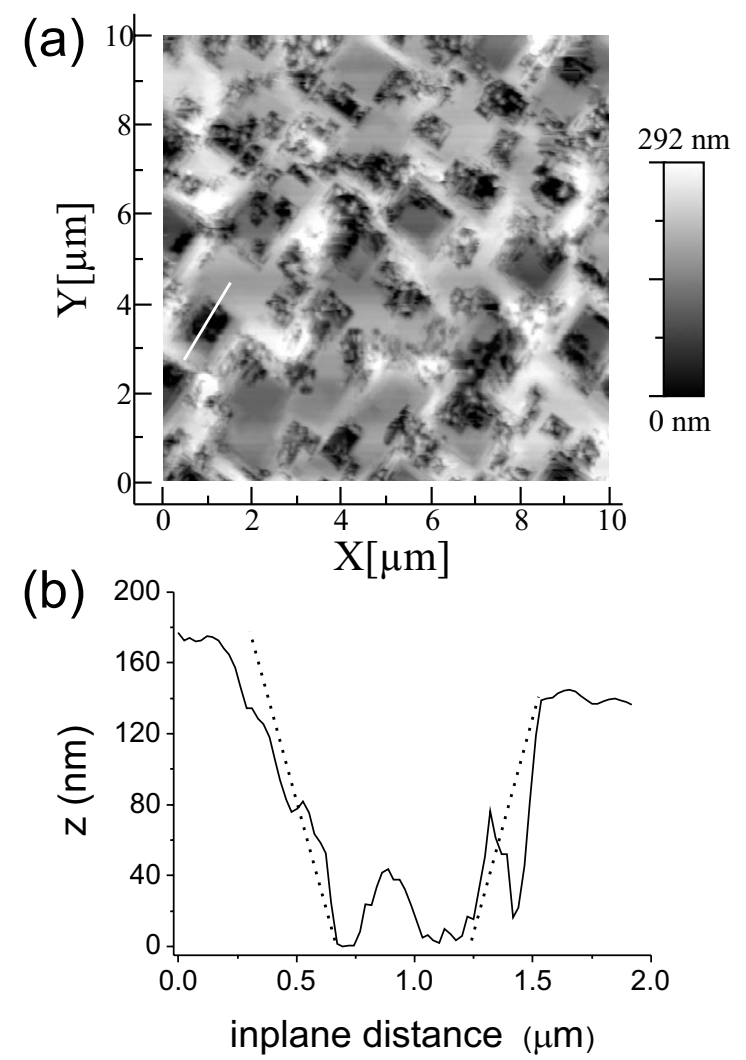

FIG. 7. (a) AFM image $(10 \times 10 \mu \mathrm{m})$ of the $\mathrm{CoGa}(100)$ surface after oxidation-induced faceting. (b) Line scan along the white line in (a). The slope of the (102) and $(10 \overline{2})$ planes is indicated by dashed lines.

dation temperature, the bulk oxide was found to form at lower oxygen partial pressures, indicative for strong kinetic barriers present during bulk oxide formation. The average oxide thickness was found to be 9 and $12 \mathrm{~nm}$ for the oxide grown at 873 and $923 \mathrm{~K}$, respectively. A diffraction signal characteristic for a truncated crystal (so-called truncation rod) without thickness oscillations was observed from the oxide film (not shown here), which points to the formation of oxide domains with large thickness variation and a locally flat surface in the order of the x-ray coherence length (several thousand angstroms).

\section{Oxygen-induced substrate faceting}

After oxidation above at a temperature of $973 \mathrm{~K}$ a qualitatively new situation was observed: the $\mathrm{CoGa}(100)$ surface undergoes a strong roughening, which was evidenced by $e x$ situ AFM investigations. Figure 7(a) shows an AFM image measured after oxidizing the sample at $1023 \mathrm{~K}$ and 5 $\times 10^{-5}$ mbar $\mathrm{O}_{2}$ for $500 \mathrm{~s}$. Under these conditions, characteristic holes by $\sim 1 \mu \mathrm{m}$ width and $\sim 100 \mathrm{~nm}$ height were observed in the AFM images. In Fig. 7(b) a line scan through one of the holes is given. The change in surface morphology was in addition characterized by in situ SXRD experiments: Fig. 8(a) shows $H$ scans parallel to the (100) substrate directions at $K=1$ and different $L$ values. Apart from the peak at $(0,1)$ due to the crystal truncation rod, additional peaks are observed as shoulders on both sides of the CTR exhibiting an
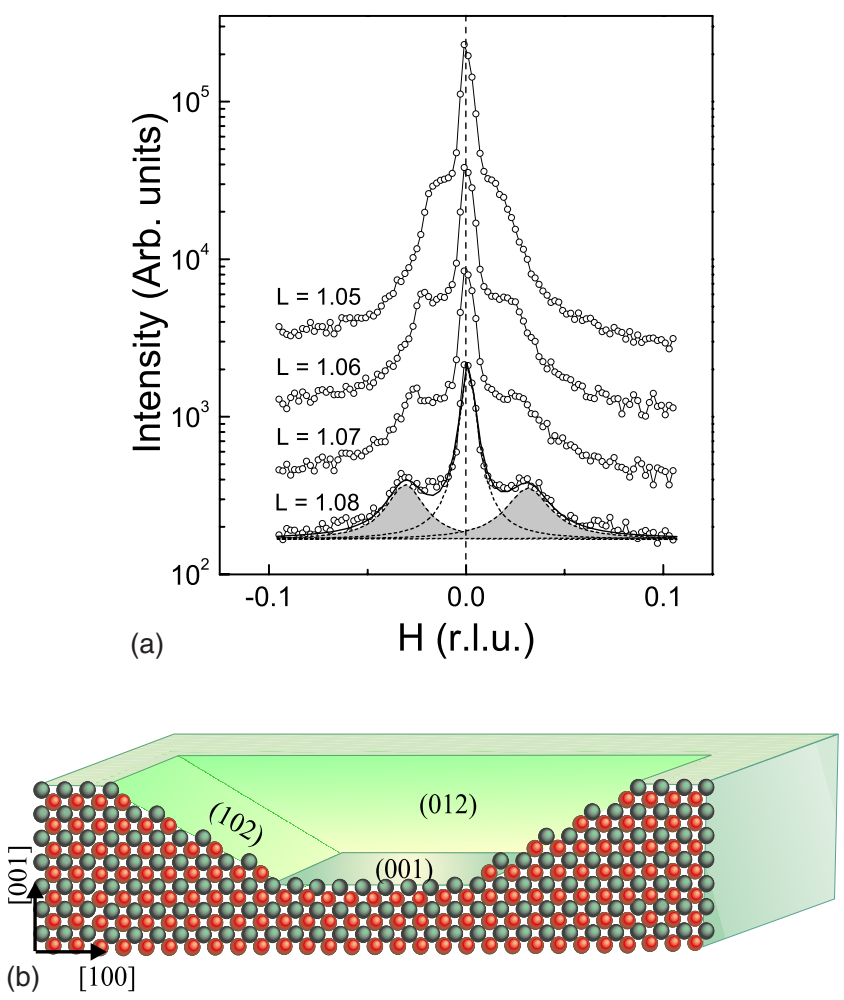

FIG. 8. (Color online) (a) Line scans performed in the $H$ direction at $K=1$ and different $L$ values. The contribution from the facets is given by the side peaks (gray), whereas the CTR signal is represented by the middle peak. (b) Schematic representation of the $(001) /\{102\}$ planes of the CoGa viewed along the [010] direction.

increase in $H$ with increasing $L$. This is a direct proof of facet formation since any facet gives rise to a crystal truncation rod signal perpendicular to its surface. In order to determine the angle between the (001) CoGa surface and the newly formed facets, each of the $H$ scans in Fig. 8(a) was fitted and the position of the side maxima was accurately determined. The angle was determined to be $26.36^{\circ} \pm 0.3^{\circ}$, corresponding to the (102)- and (102)-type facets of the substrate. The $\{102\}$ type of planes consist of two-atom-wide (101) terraces separated by monoatomic steps approximately $10 \AA$ Apart. A schematic representation of the $\mathrm{CoGa}(001) /\{102\}$ planes projected along the [010] direction is shown in Fig. 8(b). The presence of symmetry equivalent (012) and (012) facets $\left[ \pm 90^{\circ}\right.$ rotated with respect to the (102) facets] was evidenced by performing scans in the $K$ direction [not shown here] at $H=0$ at different $L$ values around the (011) substrate Bragg peak. The formation of facets with fourfold symmetry is inline with the observed squared shape of the holes visible in the AFM images. In addition, the direction of the (102) planes [plotted as dashed lines in Fig. 7(b)] agrees well with the slope of the holes, as observed by AFM.

\section{Discussion}

In the previous section we have demonstrated that the formation of the surface oxide layer takes place under quasiequilibrium conditions at an oxygen chemical potential of 


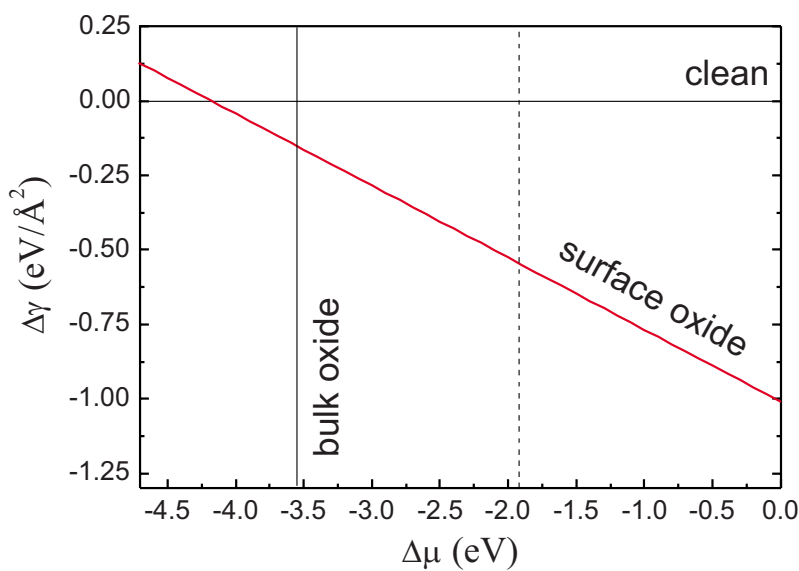

FIG. 9. (Color online) Surface phase diagram for the ultrathin Ga oxide layer on $\mathrm{CoGa}(100)$. The adhesion energy $\Delta \gamma$ of the ultrathin surface oxide as calculated from DFT is plotted as a function of the oxygen chemical potential $\Delta \mu_{\mathrm{O}}$ (solid, red line). The chemical potential for bulk oxide formation is marked as a solid vertical line and the experimentally determined chemical potential for the surface oxide formation as a dashed vertical line.

$\Delta \mu=-1.92 \mathrm{eV}$. The question arises if the surface oxide is a thermodynamically stable phase or if its growth is only possible because of the strong kinetic barriers obviously present for the bulk oxide formation over the whole pressure and temperature regime investigated in this study. The theoretical adhesion energy $\Delta \gamma$ for a surface oxide structure can be written as

$$
\Delta \gamma=\frac{1}{A}\left[E^{\text {oxide }}-\mu_{\mathrm{Ga}} N_{\mathrm{Ga}}-\mu_{\mathrm{O}} N_{\mathrm{O}}\right],
$$

where $\mu_{\mathrm{Ga}}$ and $\mu_{\mathrm{O}}$ are the chemical potentials of Ga and $\mathrm{O}$. $A$ is the surface area of the unit cell, $E^{\text {oxide }}$ is the difference of the energies with and without oxide layer, and $N_{\mathrm{Ga}}$ and $N_{\mathrm{O}}$ are the number of $\mathrm{Ga}$ and $\mathrm{O}$ atoms in the oxide unit cell; for the surface oxide $N_{\mathrm{Ga}}=N_{\mathrm{O}}=4$. In Eq. (1) the quantity $\mu_{\mathrm{Ga}}$ is not directly accessible in a simple way from the DFT calculations; nevertheless we can estimate it from (temperaturedependent) experimental values of the Ga partial Gibbs free energy of CoGa formation and the chemical potential for pure Ga (for the latter we use the DFT energy of pure Ga, $-3.52 \mathrm{eV} /$ atom, when calculating the surface oxide stability by DFT). ${ }^{33-35}$

The resulting $\Delta \gamma\left(\Delta \mu_{\mathrm{O}}\right)$ diagram is pictured in Fig. 9 for an intermediate temperature of $923 \mathrm{~K}$. The clean Coterminated CoGa surface serves as a reference with $\Delta \gamma=0$. The chemical potential for bulk oxide $\beta-\mathrm{Ga}_{2} \mathrm{O}_{3}$ formation is indicated by a solid vertical line at $\Delta \mu_{\mathrm{O}}=-3.55 \mathrm{eV}$, as it is calculated from experimental values of the heat of formation of $\beta-\mathrm{Ga}_{2} \mathrm{O}_{3}(-3.76$ eV/oxygen atom) and the $\mathrm{Ga}$ partial Gibbs free energy of CoGa formation. ${ }^{33,34,36}$ It is apparent that in this phase diagram the surface oxide is thermodynamically more stable than the clean Co-terminated surface above $\Delta \mu_{\mathrm{O}}=-4.17 \mathrm{eV}$ up to the oxygen chemical potential for bulk oxide formation. It must be noted, however, that this range is at far lower $\Delta \mu_{0}$ than accessed experimentally and we also have to expect oxygen adatom phases in this range. We have not calculated the phase stability of such phases since their detailed structure (crystal termination, oxygen sites, possible reconstruction) is unknown.

The experimental value for $\Delta \mu_{\mathrm{O}}$, above which the surface oxide is found to be stable, is indicated as a dashed vertical line in Fig. 9. It is not found in the calculated phase diagram but it is likely that its intersection with the $\Delta \gamma$ line of the surface oxide marks the transition between an unknown oxygen adsorption phase (not calculated) and the surface oxide. Obviously the surface oxide forms at an oxygen chemical potential far above the chemical potential for bulk oxide formation (note that at $923 \mathrm{~K}$ the bulk $\mathrm{Ga}_{2} \mathrm{O}_{3}$ should already form at an oxygen partial pressure of $10^{-25}$ mbar under equilibrium conditions). This implies that the surface oxide formation takes place in a metastable equilibrium with the surrounding oxygen atmosphere.

In addition, our experimental observations indicate a strong kinetic hindrance for the formation of the bulk oxide: many orders of magnitude higher oxygen pressures are needed to form the $\mathrm{Ga}_{2} \mathrm{O}_{3}$ phase, i.e., global thermodynamical equilibrium is not reached. A similar behavior was reported for the other systems, i.e., the rhodium oxide on $\operatorname{Rh}(111),{ }^{37} \sqrt{5}$ surface oxide on $\operatorname{Pd}(100),{ }^{38}$ and the ultrathin aluminum oxide on $\operatorname{NiAl}(110) .{ }^{39}$

We may speculate on the microscopic origin of such kinetic barriers. Further growth of the oxide layer may be limited by two factors: oxygen dissociation, and electron and ion transport through the growing oxide layer and by the diffusion of Ga from the substrate. The surface oxide forms a flat, two-dimensional, oxygen-terminated layer, which does not promote oxygen dissociation. Therefore, higher temperatures and oxygen pressures are needed in order to increase the probability for oxygen dissociation, e.g., at thermally induced defects. Diffusion of $\mathrm{Ga}$ through the CoGa substrate obviously does not play a role, otherwise we would not observe the formation of the Ga-rich surface oxide at temperatures much below bulk oxide formation. Thermal diffusion of ions through the oxide layer may play a role, especially for thicker oxides. The average thickness of the bulk gallium oxide was found to increase with increasing oxidation temperature ranging from 3 to $12 \mathrm{~nm}$ for temperatures between 723 and $923 \mathrm{~K}$. This is inline with an ion diffusion controlled oxidation process. ${ }^{40}$

Substrate faceting accompanies the bulk oxide formation at temperatures higher than $1023 \mathrm{~K}$. The development of a similar substrate morphology was observed after the hightemperature oxidation of NiAl (Refs. 41 and 42) and polycrystalline CoGa. ${ }^{43}$ According to Ref. 43, vacancies on the gallium sublattice develop due to the loss of gallium in the alloy surface. These vacancies are not stable and react with regular cobalt atoms to form a cobalt antisite Co defect and a cobalt vacancy on nearest-neighbor sites. If the supersaturation of defects near the surface is large enough, the formed voids may ultimately condense to macroscopic defects or cavities near the metal-oxide interface. Faceting may be also driven by the misfit between the oxide and the substrate, however, as demonstrated for PdO in Ref. 44. 


\section{CONCLUSIONS}

In conclusion, we have investigated the structure of the surface oxide grown on $\mathrm{CoGa}(100)$ and its transition to bulk $\beta-\mathrm{Ga}_{2} \mathrm{O}_{3}$ as a function of the oxidation temperature and partial oxygen pressure. An atomic level understanding of the ultrathin gallium oxide structure was achieved by means of SXRD, DFT, and STM investigations. An excellent agreement between the SXRD and STM data and the DFT-based calculations was obtained.

The stability diagram of gallium oxide on the CoGa(100) surface was mapped out by means of in situ SXRD measurements. Both a surface oxide and the bulk $\beta-\mathrm{Ga}_{2} \mathrm{O}_{3}$ phase were identified, even though, in thermodynamical equilibrium, only the bulk $\beta-\mathrm{Ga}_{2} \mathrm{O}_{3}$ is predicted to exist at any of the investigated $(p, T)$ values. The surface oxide is only metastable under the experimental conditions observed for its formation. Its structure was preserved at room temperature even at pressures as high as 1 bar $\mathrm{O}_{2}$, maintaining its high degree of crystallinity. Growth of the bulk oxide was found to be limited by kinetics, which hinder its formation for temperatures lower than $623 \mathrm{~K}$ (within the experimentally accessed time scale and pressure range). At higher temperatures, three-dimensional bulk $\mathrm{Ga}_{2} \mathrm{O}_{3}$ islands grow epitaxially on $\mathrm{CoGa}(100)$ at pressures decreasing with temperature before oxidation-induced faceting sets in at temperatures higher than $1020 \mathrm{~K}$.

This study shows that the thickness and structural perfection of the oxide layers on alloys can be tailored by the appropriate choice of oxygen pressure and temperature, which is of crucial importance for all applications involving ultrathin oxide films.

\section{ACKNOWLEDGMENTS}

We thank E. Barrena and X. Zhang for the performance of the AFM measurements. Financial support is acknowledged from the German Science Foundation in the framework of the "Graduiertenkolleg Innere Grenzflächen" (Grant No. GRK 285). The work in Vienna was supported by the Austrian Fonds zur Förderung der wissenschaftlichen Forschung.
*Corresponding author; andreas.stierle@uni-siegen.de

${ }^{\dagger}$ Present address: Universität Siegen, D-57072 Siegen, Germany.

*Present address: Leibniz Institut für innovative Mikroelektronik, D-15236 Frankfurt (Oder), Germany.

${ }^{1}$ E. Lundgren, A. Mikkelsen, J. N. Andersen, G. Kresse, M. Schmid, and P. Varga, J. Phys.: Condens. Matter 18, R481 (2006).

${ }^{2}$ J. Libuda, F. Winkelmann, M. Bäumer, H. J. Freund, T. Bertrams, H. Neddermeyer, and K. Müller, Surf. Sci. 318, 61 (1994).

${ }^{3}$ A. Stierle, F. Renner, R. Streitel, H. Dosch, W. Drube, and B. C. Cowie, Science 303, 1652 (2004).

${ }^{4}$ G. Kresse, M. Schmid, E. Napetschnig, M. Shishkin, L. Köhler, and P. Varga, Science 308, 1440 (2005).

${ }^{5}$ R. Franchy, J. Masuch, and P. Gassmann, Appl. Surf. Sci. 93, 317 (1996)

${ }^{6}$ R.-P. Blum, D. Ahlbehrendt, and H. Niehus, Surf. Sci. 396, 176 (1998).

${ }^{7}$ R.-P. Blum, D. Ahlbehrendt, and H. Niehus, Surf. Sci. 366, 107 (1996).

${ }^{8}$ M. Schmid, G. Kresse, A. Buchsbaum, E. Napetschnig, S. Gritschneder, M. Reichling, and P. Varga, Phys. Rev. Lett. 99, 196104 (2007).

${ }^{9}$ H. Graupner, L. Hammer, K. Heinz, and D. M. Zehner, Surf. Sci. 380, 335 (1997).

${ }^{10}$ G. Schmitz, P. Gassmann, and R. Franchy, J. Appl. Phys. 83, 2533 (1998).

${ }^{11}$ A. Stierle, R. Streitel, P. Nolte, A. Vlad, I. Costina, M. Marsman, G. Kresse, E. Lundgren, J. N. Andersen, R. Franchy, and H. Dosch, New J. Phys. 9, 331 (2007).

${ }^{12}$ E. Napetschnig, M. Schmid, and P. Varga, Surf. Sci. 602, 1750 (2008).

${ }^{13}$ M. Covington, J. Nowak, and D. Song, Appl. Phys. Lett. 76, 3965 (2000).
${ }^{14}$ Z. Li, C. de Groot, and J. H. Moodera, Appl. Phys. Lett. 77, 3630 (2000).

${ }^{15}$ R. Roy, V. Hill, and E. Osborn, J. Am. Chem. Soc. 74, 719 (1952).

${ }^{16}$ W. Pies and A. Weiss, in Numerical Data and Functional Relationships in Science and Technology, Landolt-Börnstein New Series, Group III Condensed Matter, Vol. 7b (Springer-Verlag, Berlin, 1975)

${ }^{17}$ J. Åhman, G. Svensson, and J. Albertsson, Acta Crystallogr., Sect. C: Cryst. Struct. Commun. 52, 1336 (1996).

${ }^{18}$ R. Franchy, Surf. Sci. Rep. 38, 195 (2000).

${ }^{19}$ R. Franchy, G. Schmitz, P. Gassmann, and F. Bartolucci, Appl. Phys. A: Mater. Sci. Process. 65, 551 (1997).

${ }^{20}$ G. Renaud, B. Villette, and P. Guénard, Nucl. Instrum. Methods Phys. Res. B 95, 422 (1995).

${ }^{21}$ E. Vlieg, J. Appl. Crystallogr. 31, 198 (1998).

${ }^{22}$ E. Vlieg, J. Appl. Crystallogr. 33, 401 (2000).

${ }^{23}$ A. Stierle, A. Steinhäuser, A. Rühm, F. U. Renner, R. Weigel, N. Kasper, and H. Dosch, Rev. Sci. Instrum. 75, 5302 (2004).

${ }^{24}$ G. Kresse and J. Furthmüller, Comput. Mater. Sci. 6, 15 (1996).

${ }^{25}$ P. E. Blöchl, Phys. Rev. B 50, 17953 (1994).

${ }^{26}$ G. Kresse and D. Joubert, Phys. Rev. B 59, 1758 (1999).

${ }^{27}$ D. M. Ceperley and B. J. Alder, Phys. Rev. Lett. 45, 566 (1980).

${ }^{28}$ J. P. Perdew and A. Zunger, Phys. Rev. B 23, 5048 (1981).

${ }^{29}$ J. Tersoff and D. R. Hamann, Phys. Rev. B 31, 805 (1985).

${ }^{30}$ J. Tersoff, Phys. Rev. B 40, 11990 (1989).

${ }^{31}$ J. Tersoff, Phys. Rev. B 41, 1235 (1990).

${ }^{32} \Delta \mu_{\mathrm{O}}(T, p)=\mu_{\mathrm{O}}^{0}\left(T, p^{0}=1\right.$ bar $)+\frac{1}{2} k_{\mathrm{B}} T \ln \left(p / p^{0}\right)$ is referenced to $1 / 2$ of the total energy of an oxygen molecule $E_{\mathrm{O}_{2}}=-5.335 \mathrm{eV}$ (DFT value): $\Delta \mu_{\mathrm{O}}(T, p)=\mu_{\mathrm{O}}(T, p)-E_{\mathrm{O}_{2}}\left[k_{\mathrm{B}}\right.$ is the Boltzmann constant, $p^{0}, \mu_{\mathrm{O}}\left(T, p^{0}=1\right.$ bar), can be found in Ref. 45].

${ }^{33}$ I. Katayama, N. Kemori, and Z. Kozuka, Trans. Jpn. Inst. Met. 16, 423 (1975).

${ }^{34}$ A. Y. Lozovoi, A. Alavi, and M. W. Finnis, Comput. Phys. Com- 
mun. 137, 174 (2001).

${ }^{35}$ Y. Jiang, J. R. Smith, and A. G. Evans, Phys. Rev. B 74, 224110 (2006).

${ }^{36}$ Scientific Group Thermodata Europe (SGTE), in Numerical Data and Functional Relationships in Science and Technology, Landolt-Börnstein New Series, Group IV Physical Chemistry, Vol. 19A, edited by I. Hurtado and D. Neuschütz (SpringerVerlag, Berlin, 2000).

${ }^{37}$ J. Gustafson, A. Mikkelsen, M. Borg, E. Lundgren, L. Köhler, G. Kresse, M. Schmid, P. Varga, J. Yuhara, X. Torrelles, C. Quirós, and J. N. Andersen, Phys. Rev. Lett. 92, 126102 (2004).

${ }^{38}$ E. Lundgren, J. Gustafson, A. Mikkelsen, J. N. Andersen, A. Stierle, H. Dosch, M. Todorova, J. Rogal, K. Reuter, and M. Scheffler, Phys. Rev. Lett. 92, 046101 (2004).
${ }^{39}$ J. P. Pierce, N. C. Bartelt, R. Stumpf, and K. F. McCarty, Phys. Rev. B 77, 195438 (2008).

${ }^{40}$ A. Stierle and H. Zabel, Europhys. Lett. 37, 365 (1997).

${ }^{41}$ J. C. Yang, K. Nadarzinski, E. Schumann, and M. Rühle, Scr. Metall. Mater. 33, 1043 (1995).

${ }^{42}$ G. Zhou and J. C. Yang, J. Mater. Res. 20, 1684 (2005).

${ }^{43}$ U. Koops and M. Martin, Solid State Ionics 136-137, 971 (2000).

${ }^{44}$ R. Westerström, J. Gustafson, A. Resta, A. Mikkelsen, J. N. Andersen, E. Lundgren, N. Seriani, F. Mittendorfer, M. Schmid, J. Klikovits, P. Varga, M. D. Ackermann, J. W. M. Frenken, N. Kasper, and A. Stierle, Phys. Rev. B 76, 155410 (2007).

${ }^{45}$ D. R. Stull and H. Prophet, JANAF Thermochemical Tables, 2nd ed. (US National Bureau of Standards, Washington, DC, 1971). 